\title{
Nonlinear RISE-Based Control of an Autonomous Underwater Vehicle
}

\author{
Nicholas Fischer, Devin Hughes, Patrick Walters, Eric M. Schwartz, Senior Member, IEEE, \\ and Warren E. Dixon, Senior Member, IEEE
}

\begin{abstract}
This study focuses on the development of a nonlinear control design for a fully-actuated autonomous underwater vehicle (AUV) using a continuous robust integral of the sign of the error control structure to compensate for system uncertainties and sufficiently smooth bounded exogenous disturbances. A Lyapunov stability analysis is included to prove semiglobal asymptotic tracking. The resulting controller is experimentally validated on an AUV developed at the University of Florida in both controlled and open-water environments.
\end{abstract}

Index Terms-Autonomous underwater vehicles (AUVs), marine robotics, nonlinear control, robust integral of the sign of the error (RISE).

\section{INTRODUCTION}

A DVANCES in sensing and control capabilities are enabling autonomous surface vehicles and autonomous underwater vehicles (AUV) to become vital assets in search and recovery, exploration, surveillance, monitoring, and military applications [1]. Accurate and robust trajectory tracking is crucial to the performance of these vehicles and advancement of autonomy in the maritime environment.

The dynamics of an AUV are time-varying, nonlinear, and often include difficult to model effects, for example, hydrodynamic coefficients and external disturbances such as sea state or ocean currents. Many results in the literature focus on AUV controllers that utilize exact knowledge of the dynamics [2]-[5].

Manuscript received April 23, 2013; revised September 9, 2013; accepted February 9, 2014. Date of publication March 14, 2014; date of current version August 4, 2014. This paper was recommended for publication by Associate Editor K. Kyriakopoulos and Editor W. K. Chung upon evaluation of the reviewers' comments.

N. Fischer, D. Hughes, and P. Walters are with the Department of Mechanical and Aerospace Engineering, University of Florida, Gainesville, FL 32611 USA (e-mail: nic.r.fischer@gmail.com; dkhughes@ufl.edu; walters8@ufl.edu).

E. M. Schwartz is with the Department of Electrical and Computer Engineering, University of Florida, Gainesville, FL 32611 USA (e-mail: ems@mil.ufl.edu).

W. E. Dixon is with the Department of Mechanical and Aerospace Engineering, University of Florida, Gainesville, FL 32611 USA and also with the Department of Electrical and Computer Engineering, University of Florida, Gainesville, FL 32611 USA (e-mail: wdixon@ufl.edu).

This paper has supplementary downloadable material available at http://ieeexplore.ieee.org, provided by the author. The video illustrates experimental validation of work that focuses on the development of a nonlinear control design for a fully-actuated autonomous underwater vehicle (AUV) using a continuous robust integral of the sign of the error (RISE) control structure to compensate for system uncertainties and sufficiently smooth bounded exogenous disturbances. The resulting controller is experimentally validated on an AUV developed at the University of Florida in both controlled and open-water environments.

Color versions of one or more of the figures in this paper are available online at http://ieeexplore.ieee.org.

Digital Object Identifier 10.1109/TRO.2014.2305791
However, while these controllers provide suitable performance and stability results in theory and simulation, obtaining empirical models of dynamic parameters (e.g., added mass, nonlinear Coriolis models, hydrodynamic damping forces, etc.) experimentally are often inaccurate and extremely difficult to develop.

Motivated to provide robustness to model uncertainty, adaptive controllers for AUVs are developed in [6]-[8]. Efforts in [9] used traditional adaptation methods and switching theory to compensate for unknown dynamics. In [10]-[16], fuzzy logic or neural network (NN)-based methods are used to approximate uncertain dynamics; however, the presence of external disturbances and function approximation errors result in uniformly ultimately bounded tracking results. An adaptive switching supervisory control technique for uncertain underactuated AUVs is developed in [17] yielding ultimately bounded tracking. Results in [14], [15], and [18] use a composite sliding mode control law to eliminate the steady state error of pure adaptive designs, in a similar manner to robust sliding mode control results in [19]-[22]. Nonlinear observers to estimate unknown hydrodynamic damping coefficients were coupled with a sliding mode control law in [23] for diving and steering control of an AUV. However, while the sliding mode technique successfully yields asymptotic tracking for uncertain nonlinear systems with additive disturbances, the resulting controller is discontinuous. Discontinuous controllers suffer from limitations such as the demand for infinite bandwidth and chatter, motivating the need for continuous methods that can yield asymptotic tracking in the presence of added disturbances and generalized uncertainty.

Motivated by our previous work in [24] and preliminary efforts in [25], a continuous robust integral of the sign of the error (RISE) control structure is used to compensate for uncertain, nonautonomous disturbances for a class of coupled, fully-actuated underwater vehicles. A Lyapunov-based stability analysis is provided to show that the control method yields semiglobal asymptotic tracking. The resulting controller is experimentally validated on a 6-degree-of-freedom (DOF) AUV that has been developed at the University of Florida. Experimental trials are conducted in a swimming pool to demonstrate the performance of the controller. Additionally, an open-water sea trial was completed in the Gulf of Mexico off the coast of Panama City Beach, FL, USA, to illustrate the robustness of the design in a real-world environment.

\section{Vehicle Configuration AND MOdELING}

The position and orientation of an AUV relative to an earthfixed frame is given by the kinematic equation of motion [26]:

$$
\dot{\eta}=J(\eta) \nu
$$




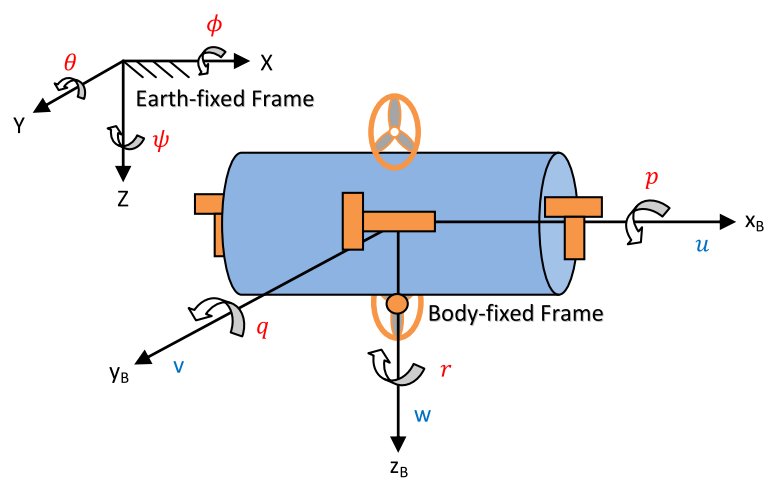

Fig. 1. AUV reference frames and associated state vector directions.

where $\nu \in \mathbb{R}^{6}$ is a vector of linear and angular velocities with coordinates in the body-fixed frame, $\eta \in \mathbb{R}^{6}$ is a vector of position and orientation with coordinates in the earth-fixed frame, and $J: \mathbb{R}^{6} \rightarrow \mathbb{R}^{6 \times 6}$ is a Jacobian transformation matrix relating the two frames. The state vectors of the AUV from (1) are illustrated in Fig. 1 and are defined as [26]

$$
\begin{aligned}
& \eta \triangleq\left[\begin{array}{llllll}
x_{\eta} & y_{\eta} & z_{\eta} & \phi_{\eta} & \theta_{\eta} & \psi_{\eta}
\end{array}\right]^{T} \\
& \nu \triangleq\left[\begin{array}{llllll}
u_{\nu} & v_{\nu} & w_{\nu} & p_{\nu} & q_{\nu} & r_{\nu}
\end{array}\right]^{T},
\end{aligned}
$$

where $x_{\eta}, y_{\eta}, z_{\eta} \in \mathbb{R}$ represent the Cartesian position of the center of mass, $\phi_{\eta}, \theta_{\eta}, \psi_{\eta} \in \mathbb{R}$ represent the orientation (roll, pitch, and yaw), $u_{\nu}, v_{\nu}, w_{\nu} \in \mathbb{R}$ represent the surge, sway, and heave velocities, and $p_{\nu}, q_{\nu}, r_{\nu} \in \mathbb{R}$ represent angular velocities. The Jacobian which relates the state vectors in (1) is defined as

$$
J(\eta) \triangleq\left[\begin{array}{cc}
J_{1}(\eta) & 0_{3 \times 3} \\
0_{3 \times 3} & J_{2}(\eta)
\end{array}\right] .
$$

In (2), $J_{1}: \mathbb{R}^{6} \rightarrow \mathbb{R}^{3 \times 3}$ and $J_{2}: \mathbb{R}^{6} \rightarrow \mathbb{R}^{3 \times 3}$ are defined as

$$
J_{1}(\eta) \triangleq
$$$$
\left[\begin{array}{ccc}
c \psi_{\eta} c \theta_{\eta} & -s \psi_{\eta} c \phi_{\eta}+c \psi_{\eta} s \theta_{\eta} s \phi_{\eta} & s \psi_{\eta} s \phi_{\eta}+c \psi_{\eta} c \phi_{\eta} s \theta_{\eta} \\
s \psi_{\eta} c \theta_{\eta} & c \psi_{\eta} c \phi_{\eta}+s \phi_{\eta} s \theta_{\eta} s \psi_{\eta} & -c \psi_{\eta} s \phi_{\eta}+s \theta_{\eta} s \psi_{\eta} c \phi_{\eta} \\
-s \theta_{\eta} & c \theta_{\eta} s \phi_{\eta} & c \theta_{\eta} c \phi_{\eta}
\end{array}\right]
$$$$
J_{2}(\eta) \triangleq\left[\begin{array}{ccc}
1 & s \phi_{\eta} t \theta_{\eta} & c \phi_{\eta} t \theta_{\eta} \\
0 & c \phi_{\eta} & -s \phi_{\eta} \\
0 & s \phi_{\eta} / c \theta_{\eta} & c \phi_{\eta} / c \theta_{\eta}
\end{array}\right],
$$

where $s \cdot, \cdot, t \cdot$ denote $\sin (\cdot), \cos (\cdot)$, and $\tan (\cdot)$, respectively, and $0_{3 \times 3} \in \mathbb{R}^{3 \times 3}$ represents a matrix of zeros.

Assumption 1: The Jacobian and its inverse exist and are bounded by a known constant $\bar{J} \in \mathbb{R}^{+}$such that $\sup _{\eta}\|J\| \leq \bar{J}$ and $\sup _{\eta}\left\|J^{-1}\right\| \leq \bar{J}$.

The definition of $J_{1}$ consists of a sequence of three rotations about each of the primary orientation axes. The order in which these rotations is completed is not arbitrary; however, for guidance and control applications, it is common to use the $x y z$-convention in terms of Euler angles. Utilizing this convention, $J_{2}$ is undefined for a pitch angle of $\theta_{\eta}= \pm 90^{\circ}$, which violates Assumption 1. However, during routine "flight" operations with underwater vehicles, the parameter regions $\theta_{\eta}= \pm 90^{\circ}$ are unlikely due to metacentric restoring forces [26]. To handle situations when operation near $\theta_{\eta}= \pm 90$ is required, additional supplemental techniques can be used to modify the reference coordinate system that is used to define the order of the Euler angles when the vehicle is nearing a singular orientation. An arbitration algorithm must be used to correctly manage the the transition periods when the coordinate system convention is redefined.

Under assumptions that 1) the body-fixed frame coincides with the center of mass of the AUV, 2) accelerations of a point on the surface of the earth can be neglected (i.e., the reference frame XYZ in Fig. 1 is considered to be inertial), and 3) added mass is constant (independent of wave frequency), the dynamic motion of the AUV can be described by a body-fixed vector representation as [26]

$$
M \dot{\nu}+C(\nu) \nu+D(\nu) \nu+g(\eta)+\tau_{d}=\tau_{b}
$$

where $M \in \mathbb{R}^{6 \times 6}$ denotes inertia (including added mass), $C$ : $\mathbb{R}^{6} \rightarrow \mathbb{R}^{6 \times 6}$ denotes Coriolis and centripetal effects (including added mass), $D: \mathbb{R}^{6} \rightarrow \mathbb{R}^{6 \times 6}$ denotes hydrodynamic damping effects, $g: \mathbb{R}^{6} \rightarrow \mathbb{R}^{6}$ is the vector of hydrostatic (gravitational and buoyancy) forces and moments, $\tau_{d} \in \mathbb{R}^{6}$ is a vector of nonlinear disturbances (e.g., current, waves, tether forces, etc.), and $\tau_{b} \in \mathbb{R}^{6}$ is a vector of external forces and moments about the center of mass in the body-fixed frame. An earth-fixed representation of the dynamics [26] can be generated by applying the kinematic transformations in (1) to (3) to obtain

$$
\bar{M}(\eta) \ddot{\eta}+\bar{C}(\eta, \dot{\eta}, \nu) \dot{\eta}+\bar{D}(\eta, \nu) \dot{\eta}+\bar{g}(\eta)+\bar{\tau}_{d}=\tau_{n}
$$

where $\bar{M} \triangleq J^{-T} M J^{-1}, \bar{C} \triangleq J^{-T}\left[C-M J^{-1} \dot{J}\right] J^{-1}, \bar{D} \triangleq$ $J^{-T} D J^{-1}, \bar{g} \triangleq J^{-T} g, \bar{\tau}_{d} \triangleq J^{-T} \tau_{d}$, and $\tau_{n} \triangleq J^{-T} \tau_{b}$. The subsequent development is based on the assumptions that $\eta$ and $\nu$ are measurable (using sensors common to many AUVs as discussed in [27]) and that $\bar{M}, \bar{C}, \bar{D}, \bar{g}$, and $\bar{\tau}_{d}$ are unknown functions. ${ }^{1}$

Assumption 2: The disturbance term and its first two time derivatives are bounded, i.e., $\tau_{d}, \dot{\tau}_{d}, \ddot{\tau}_{d} \in \mathcal{L}_{\infty}$. ${ }^{2}$

Assumption 3: The desired trajectory $\eta_{d} \in \mathbb{R}^{n}$ is designed such that $\eta_{d}^{(i)} \in \mathbb{R}^{n}, \forall i=0,1, \ldots, 4$ exist and are bounded. ${ }^{3}$

Property 1: The inertia matrix $\bar{M}$ is symmetric, positive definite, and satisfies the following inequality $\forall \xi \in \mathbb{R}^{n}, \eta \in \mathbb{R}^{6}$ :

$$
\underline{m}\|\xi\|^{2} \leq \xi^{T} \bar{M}(\eta) \xi \leq \bar{m}(\eta)\|\xi\|^{2}
$$

where $\underline{m} \in \mathbb{R}^{+}$is a known constant, and $\bar{m}: \mathbb{R}^{6} \rightarrow \mathbb{R}^{+}$is a known function.

Property 2: The dynamics terms $\bar{C}, \bar{D}$, and $\bar{g}$ are bounded provided $\eta$ and $\nu$ are bounded.

\footnotetext{
${ }^{1}$ Structural or measurable knowledge of the unknown terms $\bar{M}, \bar{C}, \bar{D}, \bar{g}$ allow the user to more appropriately define upper bounds such that the gain conditions presented in Theorem 1 can be satisfied.

${ }^{2}$ Many practical disturbance terms satisfy this assumption including wind disturbances, wave disturbances, ocean currents, etc. when simple bounding assumptions are imposed (e.g., bounded current velocity, bounded wind velocity, etc.) [26], [28], [29].

${ }^{3}$ Many guidance and navigation applications utilize smooth high-order differentiable desired trajectories. Curve fitting or nonlinear filter methods can be used to generate sufficiently smooth time-varying trajectories.
} 


\section{CONTROL DeVElopMENT}

\section{A. Error Systems}

The objective is to design a controller that ensures the system state tracks a sufficiently smooth desired trajectory. To quantify the control objective, a tracking error $e_{1} \in \mathbb{R}^{6}$ is defined as

$$
e_{1} \triangleq \eta_{d}-\eta
$$

Two auxiliary tracking errors $e_{2}, r \in \mathbb{R}^{6}$ are defined as

$$
\begin{gathered}
e_{2} \triangleq \dot{e}_{1}+\alpha_{1} e_{1} \\
r \triangleq \dot{e}_{2}+\alpha_{2} e_{2}
\end{gathered}
$$

where $\alpha_{1}, \alpha_{2} \in \mathbb{R}^{+}$are constant gains. The auxiliary signal $r$ is introduced to facilitate the stability analysis and is not used in the control design since the expression in (7) depends on the unmeasurable state $\ddot{\eta}$.

\section{B. Open-Loop Error System}

Premultiplying (7) by $\bar{M}(\eta)$ and using (4)-(6), the open-loop error system can be expressed as

$$
\bar{M}(\eta) r=f_{d}+S+\bar{\tau}_{d}-\tau_{n}
$$

where the auxiliary function $f_{d} \in \mathbb{R}^{6}$ is defined as

$$
f_{d} \triangleq \bar{M}\left(\eta_{d}\right) \ddot{\eta}_{d}+\bar{C}\left(\eta_{d}, \dot{\eta}_{d}, \nu_{d}\right) \dot{\eta}_{d}+\bar{D}\left(\eta_{d}, \nu_{d}\right) \dot{\eta}_{d}+g\left(\eta_{d}\right)
$$

the desired signal $\nu_{d}$ can be determined using the known kinematic relationship in (1) and the known signals $\eta_{d}$ and $\dot{\eta}_{d}$, and the auxiliary function $S \in \mathbb{R}^{6}$ is defined as

$$
\begin{aligned}
S= & \bar{M}(\eta) \ddot{\eta}+\bar{C}(\eta, \dot{\eta}, \nu) \dot{\eta}+\bar{D}(\eta, \nu) \dot{\eta} \\
& +\bar{g}(\eta)-f_{d}+\alpha_{2} \dot{e}_{1}+\alpha_{2} e_{2} .
\end{aligned}
$$

Strategic grouping allows terms included in $f_{d}$ to be upper bounded by constants since they are all functionally dependent on bounded trajectories. The remaining terms in $S$ are subsequently bounded by a state-dependent function for use in the analysis, as shown in the next section.

\section{Control Design}

From (8), the controller is designed using a RISE feedback structure as [30], [31]

$$
\tau_{n} \triangleq\left(k_{s}+1\right) e_{2}-\left(k_{s}+1\right) e_{2}(0)+v_{F}
$$

where $v_{F} \in \mathbb{R}^{6}$ is the Filippov solution to the following differential equation

$$
\dot{v}_{F} \triangleq\left(k_{s}+1\right) \alpha_{2} e_{2}+\beta \operatorname{sgn}\left(e_{2}\right), v_{F}(0)=0
$$

$\beta, k_{s} \in \mathbb{R}$ are positive, constant control gains, and $\operatorname{sgn}(\cdot)$ is defined $\forall \xi \in \mathbb{R}^{m}=\left[\begin{array}{llll}\xi_{1} & \xi_{2} & \cdots & \xi_{m}\end{array}\right]^{T}$ as $\operatorname{sgn}(\xi) \triangleq$ $\left[\begin{array}{llll}\operatorname{sgn}\left(\xi_{1}\right) & \operatorname{sgn}\left(\xi_{2}\right) & \ldots & \operatorname{sgn}\left(\xi_{m}\right)\end{array}\right]^{T}$. The differential equation given in (10) is continuous except when $e_{2}=0$. Using

${ }^{4}$ RISE feedback structure can be coupled with a feedforward term, i.e., NNs [24], [25], model-based adaptive law [30] or known dynamics, for improved performance.
Filippov's theory of differential inclusions [32]-[35], the existence of solutions can be established for $\dot{v}_{F} \in K\left[h_{1}\right]\left(e_{2}\right)$, where $h_{1}: \mathbb{R}^{6} \rightarrow \mathbb{R}^{6}$ is defined as the right-hand side (RHS) of (10) and $K\left[h_{1}\right] \triangleq \bigcap_{\delta>0} \bigcap_{\mu S_{m}=0} \overline{c o} h_{1}\left(B\left(e_{2}, \delta\right) \backslash S_{m}\right)$, where $\bigcap_{\mu S_{m}=0}$ denotes the intersection of all sets $S_{m}$ (of Lebesgue measure zero) of discontinuities, $\overline{c o}$ denotes convex closure, and $B\left(e_{2}, \delta\right) \triangleq\left\{\varsigma \in \mathbb{R} \mid\left\|e_{2}-\varsigma\right\|<\delta\right\}$ [36], [37].

Remark 1: Typical control techniques that can achieve asymptotic convergence in the presence of a disturbance either utilize discontinuous feedback or feedback with a discontinuous derivative. Continuously differentiable robust techniques such as high-gain feedback can only achieve uniformly ultimately bounded convergence. Discontinuous control techniques (such as sliding mode or variable structure control) suffer from limitations such as demand for infinite bandwidth or chatter. Because the controller in (9) utilizes the integral of a discontinuous signal, the implemented control law does not suffer from these restrictions, while still compensating for sufficiently smooth nonlinear disturbances and system uncertainties.

\section{Closed-Loop Error System}

To facilitate the subsequent stability analysis, the controller in (9) is substituted into (8) and the time derivative is determined as

$$
\begin{aligned}
\bar{M}(\eta) \dot{r}= & -\frac{1}{2} \dot{\bar{M}}(\eta, \dot{\eta}) r+\tilde{N}+N_{d} \\
& -e_{2}-\left(k_{s}+1\right) r-\beta \operatorname{sgn}\left(e_{2}\right)
\end{aligned}
$$

where $\tilde{N} \in \mathbb{R}^{6}$ and $N_{d} \in \mathbb{R}^{6}$ are defined as

$$
\begin{aligned}
& \tilde{N} \triangleq-\frac{1}{2} \dot{\bar{M}}(\eta, \dot{\eta}) r+\dot{S}+e_{2} \\
& N_{d} \triangleq \dot{f}_{d}+\dot{\tau}_{d} .
\end{aligned}
$$

Since $\bar{C}$ is not skew symmetric in the earth-fixed representation of the dynamics in (4), a portion of the $\dot{\bar{M}}$ term is included in the closed-loop error system to help cancel cross terms in the stability analysis, while the remainder of the term is placed inside the $\tilde{N}$ term. Using (5)-(6) and the Mean Value Theorem, the function $\tilde{N}$ in (12) can be upper bounded as [38, App. A]

$$
\|\tilde{N}\| \leq \rho(\|z\|)\|z\|
$$

where $z \in \mathbb{R}^{18}$ is defined as

$$
z \triangleq\left[\begin{array}{lll}
e_{1}^{T} & e_{2}^{T} & r^{T}
\end{array}\right]^{T}
$$

and $\rho: \mathbb{R}^{+} \rightarrow \mathbb{R}^{+}$is a positive, globally invertible function. From Assumptions 2 and 3, the following inequalities can be developed:

$$
\left\|N_{d}\right\| \leq \zeta_{1}, \quad\left\|\dot{N}_{d}\right\| \leq \zeta_{2}
$$

where $\zeta_{1}, \zeta_{2} \in \mathbb{R}^{+}$are known constants.

\section{Stability Analysis}

Theorem 1: The controller in (9)-(10) ensures that the states and controller are bounded and the tracking errors are regulated 


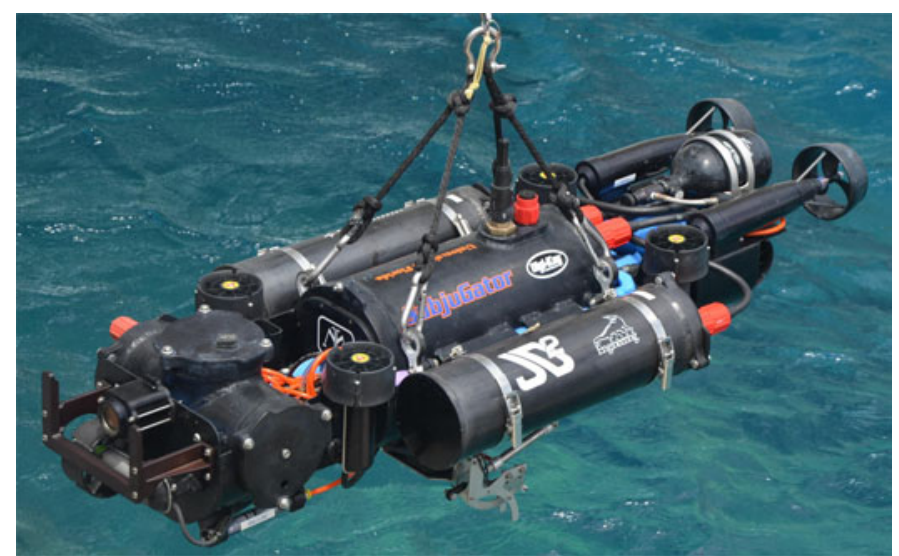

Fig. 2. AUV, "SubjuGator 7," developed at the University of Florida.

in the sense that

$$
\left\|e_{1}\right\| \rightarrow 0 \quad \text { as } \quad t \rightarrow \infty
$$

provided $k_{s}$ is selected sufficiently large based on the initial conditions of the states, and the remaining control gains are selected based on the following sufficient conditions:

$$
\beta>\zeta_{1}+\frac{1}{\alpha_{2}} \zeta_{2}, \quad \alpha_{1}>\frac{1}{2}, \quad \alpha_{2}>\frac{1}{2} .
$$

Proof: See the Appendix

\section{EXPERIMENTAL RESULTS}

\section{A. Vehicle Configuration}

The validation experiments are completed using an AUV (known as "SubjuGator 7") that has been developed at the University of Florida. The AUV, shown in Fig. 2, is a hybrid AUV that is designed with emphasis on modularity and fault tolerance. The vehicle has physical dimensions of $1.3 \mathrm{~m} \times 0.5 \mathrm{~m}$ $\times 0.5 \mathrm{~m}$ and is configured with eight bidirectional thrusters in a redundant configuration with four heave thrusters, two sway thrusters, and two primary surge thrusters, allowing for maneuvering in $6 \mathrm{DOF}$. The relationship between the force/moment acting on the vehicle and the control input of each individual thruster can be described by a thruster mapping algorithm, such as the one described in [39].

The vehicle utilizes specialized independent pressure vessels to house core computing functions. Motor controllers, networking capabilities, and platform-specific processing are located in a central pressure vessel. This vessel also houses processing of external sensors (e.g., cameras, sonars, etc.) utilizing a 2.13-GHz Quad-core Xeon processor. Navigation and control capabilities are located in a separate T-shaped navigation pressure vessel at the front of the vehicle. The navigation vessel includes: vehicle-independent navigation sensors (e.g., inertial measurement unit (IMU), Doppler velocity log (DVL), depth sensor, temperature sensor, and GPS receiver) and the processing capability to unify the data sources. On-board localization, navigation, and control are computed in the navigation vessel on a $720-\mathrm{MHz}$ OMAP processor.

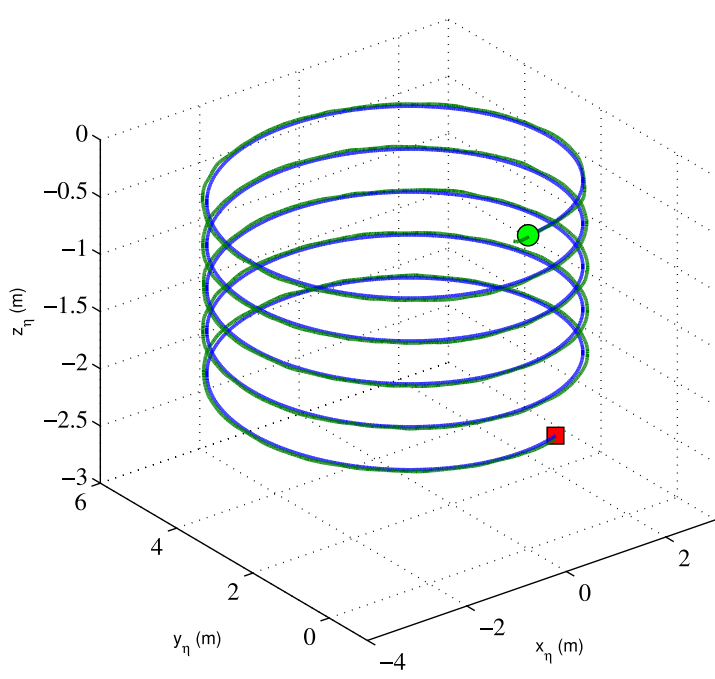

Fig. 3. Time history of the position of the AUV with respect to the helical trajectory.

To localize the state of the vehicle, an indirect unscented Kalman filter estimates the error in position, velocity, and orientation generated by the inertial navigation system (INS), which includes high-speed sensory inputs $(205 \mathrm{~Hz})$ from an analog device ADIS16405 9-DOF IMU (providing triaxis magnetometer, accelerometer, and gyroscopic inputs). Input error signals for the Kalman filter are generated using low-speed reference sensors: a Teledyne Explorer DVL (providing threeaxis velocity and height over bottom), a 14-channel GPS receiver (while surfaced), a 10-bar pressure sensor, and a filtered tilt/magnetometer/gravity-based estimation of attitude. Mission commands are executed by interpreting information from onboard optical sensors and are then converted to sufficiently smooth desired vehicle trajectories that are based on the dynamic capabilities of the vehicle. By computing smooth desired trajectories from waypoints in real time, the controller avoids large steps in error (potentially producing large actuation efforts and posing risks of actuator saturation). State estimation and control loops for the vehicle are sampled at $50 \mathrm{~Hz}$.

\section{B. Controlled Environment Study}

1) Experimental Setup: The first set of experiments were performed in a swimming pool at the University of Florida under the influence of parametric system uncertainties and small unknown disturbance effects such as pump currents and tether forces. For this enclosed space, the vehicle is commanded to track a helical desired trajectory beginning from a depth of approximately $0.25 \mathrm{~m}$, traveling to a depth of $2.25 \mathrm{~m}$. Possible kinematic singularities are avoided by choosing a desired trajectory that remains sufficiently far from pitch angles of $\pm 90^{\circ}$, and the vehicle is designed such that metacentric restoring forces help regulate the pitch and roll of the vehicle.

2) Results: Fig. 3 depicts the time history of the vehicle in the inertial frame. The circle denotes the starting coordinate and the square denotes the goal coordinate. Tracking errors for the inertial position and orientation are shown in Fig. 4. The errors 

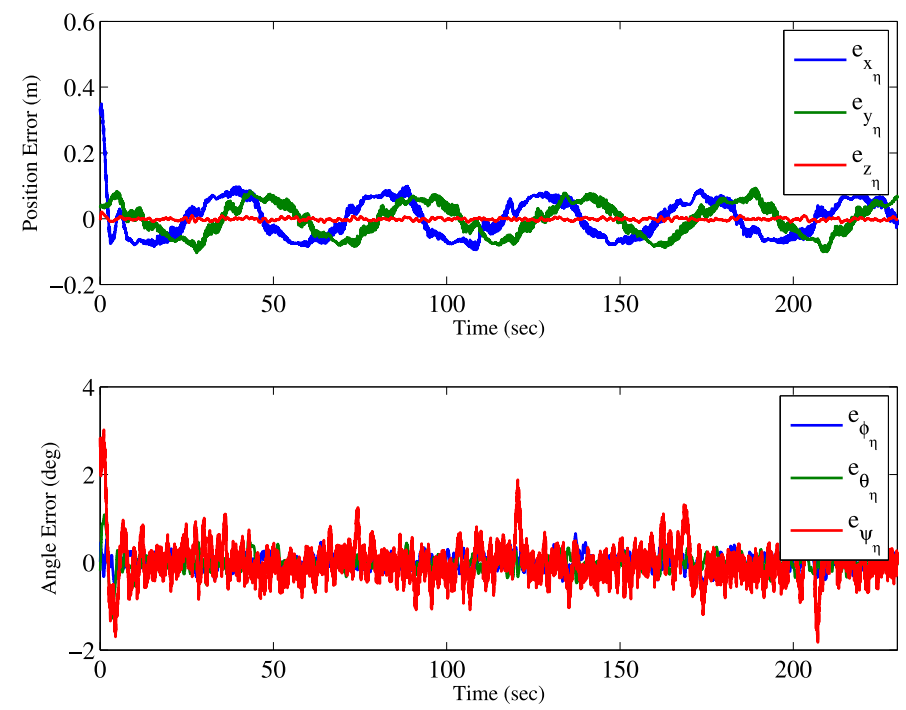

Fig. 4. Tracking errors for the inertial position (top) and attitude (bottom) of the vehicle.

TABLE I

RMS TRACKING ERRORS FROM THE CONTROLLED ENVIRONMENT AND OPEN-WATER STUDIES

\begin{tabular}{|c|c|c|c|}
\hline \multirow{4}{*}{ 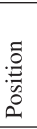 } & \multicolumn{2}{|r|}{ Open-Water } & Pool \\
\hline & $x_{\eta_{r m s}}$ & $0.0866 \mathrm{~m}$ & $0.0594 \mathrm{~m}$ \\
\hline & $y_{\eta_{r m s}}$ & $0.0916 \mathrm{~m}$ & $0.0495 \mathrm{~m}$ \\
\hline & $z_{\eta_{r m s}}$ & $0.0626 \mathrm{~m}$ & $0.0042 \mathrm{~m}$ \\
\hline \multirow{3}{*}{ 总 } & $\phi_{\eta_{r m s}}$ & $1.0048^{\circ}$ & $0.1576^{\circ}$ \\
\hline & $\theta_{\eta_{r m s}}$ & $1.4559^{\circ}$ & $0.1915^{\circ}$ \\
\hline & $\psi_{\eta_{r m s}}$ & $2.7252^{\circ}$ & $0.4507^{\circ}$ \\
\hline
\end{tabular}

are also numerically represented in Table I. The control forces and moments about the center of mass of the vehicle are shown in Fig. 5.

Results can be viewed in the video accompanying this paper at the following url: http://ncr.mae.ufl.edu/index.php? $\mathrm{id}=$ research/sub_RISE.

\section{Open-Water Study}

1) Experimental Setup: An open-water sea trial was completed in the Gulf of Mexico off the coast of Panama City Beach, FL, USA, to analyze and compare the effectiveness of the controller in a real-world environment. The study was completed in shallow water (approximately 5-m deep), with wave heights of approximately $0.5-1.25 \mathrm{~m}$ and a measured current of $0.08 \mathrm{~m} / \mathrm{s}$. Gulf currents and surface effects are considered unknown disturbances to the system, and all coefficients of the AUV's dynamic model (i.e., inertia, hydrodynamic forces, etc.) are uncertain. The experiment considers a continuous linear search pattern that is converted in real time to smooth $\mathcal{C}^{4}$ trajectories; the pattern consists of segments 2 and $10 \mathrm{~m}$ in length. Beginning at the surface, the vehicle submerges to a depth of $2 \mathrm{~m}$ and follows the linear segments. Possible kinematic singularities are avoided by choosing a desired trajectory that remains
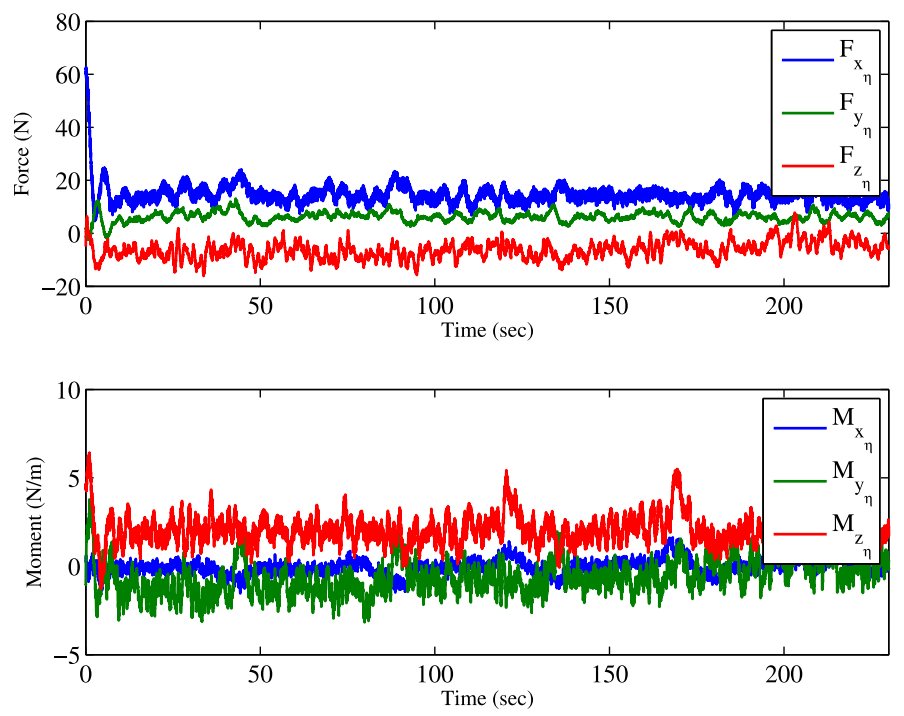

Fig. 5. Control efforts commanded about the center of mass of the vehicle.

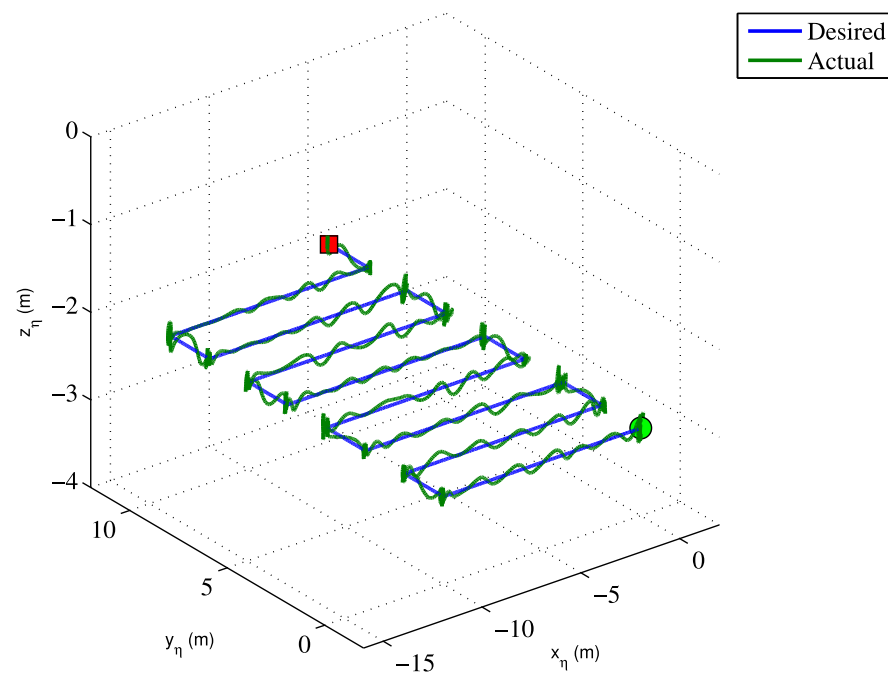

Fig. 6. Time history of the position of the AUV with respect to a linear search pattern for the RISE controller.

sufficiently far from pitch angles of $\pm 90^{\circ}$, and the vehicle is designed such that metacentric restoring forces help regulate the pitch and roll of the vehicle.

2) Results: Fig. 6 illustrates the RISE controller in threedimensional space as a function of time as compared with the commanded desired trajectory. Fig. 7 illustrates the position errors in meters and the attitude errors in degrees for the RISE controller. The errors are also numerically represented in Table I. Fig. 8 illustrates the control efforts provided for each controller.

Because of the shallow water trajectory, wave interactions and surface effects are apparent in the depth positioning of the AUV as illustrated in Fig. 6. However, the AUV still maintains accurate tracking of the trajectory despite these disturbances.

The experimental results demonstrate that the control strategy provides robustness to both parametric uncertainty and 

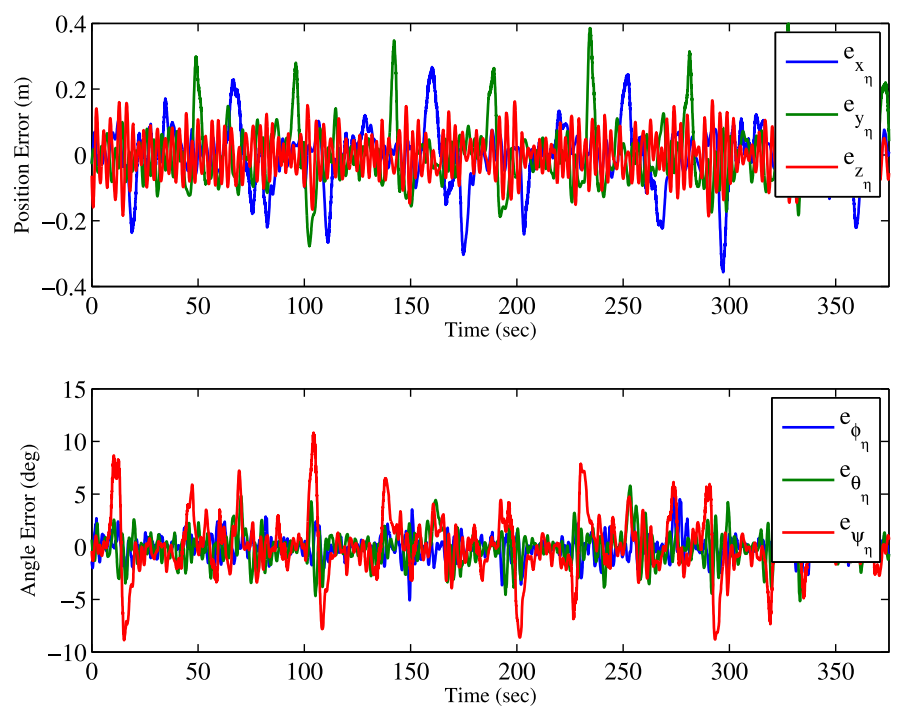

Fig. 7. Inertial position errors (top) and attitude errors (bottom) for the RISE controller.
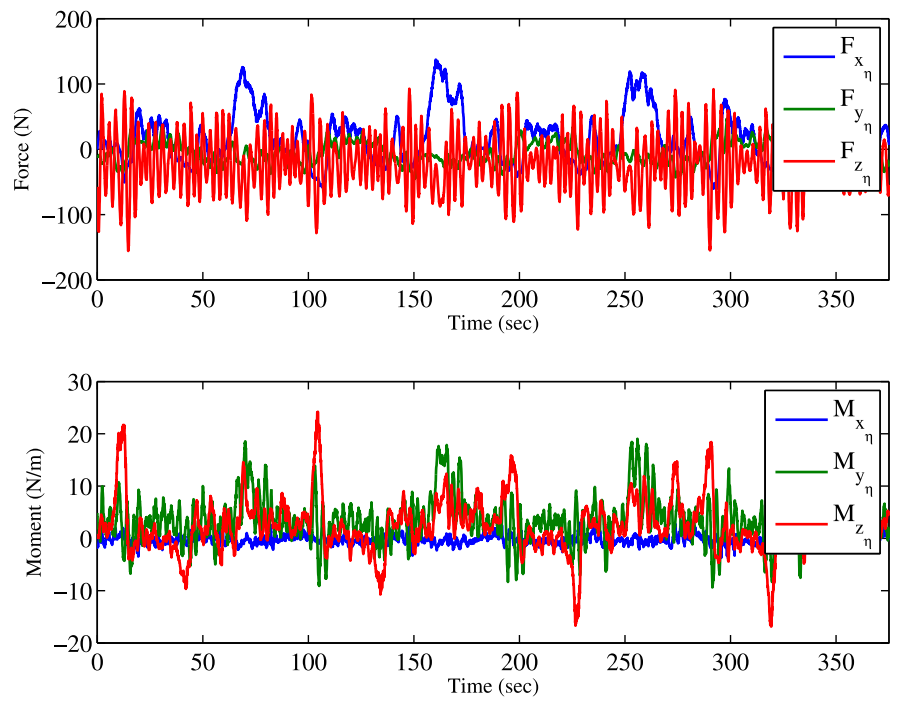

Fig. 8. Control effort forces (top) and moments (bottom) about the center of mass for the RISE controller.

measurement noise. The use of industry-standard navigation sensors (MEMs-based IMU and DVL) illustrate the utility of the control method on real-world systems.

\section{CONCLUSION}

A continuous robust controller has been developed and experimentally validated for an AUV. The control scheme compensates for complete model uncertainty yielding semiglobal asymptotic tracking. The control design is implemented on an AUV in both controlled and open-water environments to illustrate the performance of the controller on a physical system. Despite larger orientation errors in the open-water study, the per- formance is well within the desired performance level to operate the vehicle. With kinematic modifications, the RISE-based feedback control law can also be applied to slender-bodied AUVs.

\section{APPENDIX}

\section{PROOF OF THEOREM 1}

Proof: Let $y \in \mathbb{R}^{19}$ be defined as

$$
y \triangleq\left[\begin{array}{ll}
z^{T} & \sqrt{P}
\end{array}\right]^{T} .
$$

In (17), the auxiliary function $P \in \mathbb{R}$ is defined as the Filippov solution to the following differential equation:

$$
\begin{aligned}
\dot{P} & =-r^{T}\left(N_{d}-\beta \operatorname{sgn}\left(e_{2}\right)\right) \\
P\left(t_{0}\right) & =\beta \sum_{i=1}^{n}\left|e_{2_{i}}\left(t_{0}\right)\right|-e_{2}\left(t_{0}\right)^{T} N_{d}\left(t_{0}\right)
\end{aligned}
$$

where the subscript $i=1,2, \ldots, n$ denotes the $i$ th element of the vector, and $\beta$ is chosen according to the sufficient conditions in (16). Similar to the development in (10), existence of solutions for $P$ can be established using Filippov's theory of differential inclusions for $\dot{P} \in K\left[h_{2}\right]\left(e_{2}, r, t\right)$, where $h_{2}: \mathbb{R}^{12} \times \mathbb{R}^{+} \rightarrow$ $\mathbb{R}^{+}$is defined as $h_{2} \triangleq-r^{T}\left(N_{d}-\beta \operatorname{sgn}\left(e_{2}\right)\right)$ and $K\left[h_{2}\right] \triangleq$ $\bigcap_{\delta>0} \bigcap_{\mu S_{m}=0} \overline{c o} h_{2}\left(B\left(e_{2}, \delta\right) \backslash S_{m}, r, t\right)$ as in (10). Integrating (18) by parts and provided the sufficient conditions in (16) are satisfied, $P \geq 0$ (see [24] for details).

Let $\mathcal{D} \triangleq\left\{y \in \mathbb{R}^{19} \mid\|y\| \leq \rho^{-1}\left(2 \sqrt{\lambda_{1} k_{s}}\right)\right\}$ be an open and connected set where $\lambda_{1}=\min \left\{\alpha_{1}-\frac{1}{2}, \alpha_{2}-\frac{1}{2}, 1\right\}$, and $V_{L}$ : $\mathcal{D} \times[0, \infty) \rightarrow \mathbb{R}$ be continuously differentiable in $y$, locally Lipschitz in $t$, regular and defined as

$$
V_{L}(y, t)=\frac{1}{2} e_{1}^{T} e_{1}+\frac{1}{2} e_{2}^{T} e_{2}+\frac{1}{2} r^{T} \bar{M}(\eta(t)) r+P
$$

which satisfies the following inequalities:

$$
U_{1}(y) \leq V_{L}(y, t) \leq U_{2}(y)
$$

where $U_{1}: \mathbb{R}^{19} \rightarrow \mathbb{R}$ and $U_{2}: \mathbb{R}^{19} \rightarrow \mathbb{R}$ are positive definite functions defined as $U_{1} \triangleq \frac{1}{2} \min \{1, m\}\|y\|^{2}$ and $U_{2} \triangleq$ $\max \left\{\frac{1}{2} \bar{m}(\eta), 1\right\}\|y\|^{2}$.

Under Filippov's framework, strong stability of the closedloop system $\dot{y}=h_{3}(y, t)$ can be established, where $h_{3}: \mathbb{R}^{19} \times$ $\mathbb{R}^{+} \rightarrow \mathbb{R}^{19}$ denotes the RHS of the closed-loop error signals. The time derivative of (19) exists almost everywhere (a.e.), i.e., for almost all $t \in \mathbb{R}^{+}$, and $\dot{V}_{L}(y(t), t) \stackrel{a . e .}{\in} \dot{\tilde{V}}_{L}(y(t), t)$ where

$$
\dot{\tilde{V}}_{L}=\bigcap_{\xi \in \partial V_{L}(y, t)} \xi^{T} K\left[\begin{array}{lllll}
\dot{e}_{1}^{T} & \dot{e}_{2}^{T} & \dot{r}^{T} & \frac{1}{2} P^{-\frac{1}{2}} \dot{P} & 1
\end{array}\right]^{T}
$$

where $\partial V_{L}$ is the generalized gradient of $V_{L}$ [36], [37], [40]. Since $V_{L}$ is continuously differentiable with respect to $y$

$$
\dot{\tilde{V}}_{L} \subset \nabla V_{L}^{T} K\left[\begin{array}{lllll}
\dot{e}_{1}^{T} & \dot{e}_{2}^{T} & \dot{r}^{T} & \frac{1}{2} P^{-\frac{1}{2}} \dot{P} & 1
\end{array}\right]^{T}
$$

where $\nabla V_{L} \triangleq\left[\begin{array}{lllll}e_{1}^{T} & e_{2}^{T} & r^{T} \bar{M} & 2 P^{\frac{1}{2}} & \frac{1}{2} r^{T} \dot{\bar{M}} r\end{array}\right]^{T}$.

Using the calculus for $K[\cdot]$ from [37], substituting (5), (6), (9), (11), and (18), and canceling similar terms, the expression 
in $(21)$ becomes

$$
\begin{aligned}
\dot{\tilde{V}}_{L} \subset & e_{1}^{T} e_{2}-\alpha_{1} e_{1}^{T} e_{1}-\alpha_{2} e_{2}^{T} e_{2}+r^{T} \tilde{N}+r^{T} N_{d} \\
& -\left(k_{s}+1\right) r^{T} r-r^{T} \beta K\left[\operatorname{sgn}\left(e_{2}\right)\right] \\
& -r^{T}\left(N_{d}-\beta K\left[\operatorname{sgn}\left(e_{2}\right)\right]\right)
\end{aligned}
$$

where $K\left[\operatorname{sgn}\left(e_{2}\right)\right]=\operatorname{SGN}\left(e_{2}\right)$ [37] such that $\operatorname{SGN}\left(e_{2_{i}}\right)=1$ if $e_{2_{i}}>0,[-1,1]$ if $e_{2_{i}}=0$, and -1 if $e_{2_{i}}<0 .{ }^{5}$ Utilizing the fact that the set in (22) reduces to a scalar equality since the RHS is continuous a.e., i.e., the RHS is continuous except for the Lebesgue negligible set of times when $r^{T} \beta K\left[\operatorname{sgn}\left(e_{2}\right)\right]-$ $r^{T} \beta K\left[\operatorname{sgn}\left(e_{2}\right)\right] \neq 0^{6}$ [36], [44], an upper bound for $\dot{V}_{L}$ is given as

$$
\begin{gathered}
\dot{V}_{L} \stackrel{\text { a.e. }}{\leq}-\alpha_{1}\left\|e_{1}\right\|^{2}+\left\|e_{1}\right\|\left\|e_{2}\right\|-\alpha_{2}\left\|e_{2}\right\|^{2} \\
+\rho(\|z\|)\|r\|\|z\|-\left(k_{s}+1\right)\|r\|^{2} .
\end{gathered}
$$

Utilizing Young's Inequality, the expression in (23) can be reduced to

$$
\dot{V}_{L} \stackrel{\text { a.e. }}{\leq}-\lambda_{1}\|z\|^{2}-k_{s}\|r\|^{2}+\rho(\|z\|)\|r\|\|z\|
$$

where $z$ was defined in (15). Provided the sufficient conditions in (16) are satisfied, and by completing the squares for $r$, the expression in (24) can be upper bounded as

$$
\dot{V}_{L} \stackrel{\text { a.e. }}{\leq}-\lambda_{1}\|z\|^{2}+\frac{\rho^{2}(\|z\|)\|z\|^{2}}{4 k_{s}} \leq-U(y)
$$

where $U: \mathbb{R}^{19} \rightarrow \mathbb{R}$ is positive definite function defined as $U \triangleq$ $c\|z\|^{2}$, for some positive constant $c \in \mathbb{R}$.

The inequalities in (20) and (25) can be used to show that $V_{L} \in$ $\mathcal{L}_{\infty}$, thus, $e_{1}, e_{2}, r, P \in \mathcal{L}_{\infty}$. Given that $e_{1}, e_{2} \in \mathcal{L}_{\infty}$, standard linear analysis can be used to show that $\dot{e}_{1}, \dot{e}_{2} \in \mathcal{L}_{\infty}$ from (6) and Assumption 1. Since $e_{1}, e_{2}, r \in \mathcal{L}_{\infty}$ and $\eta_{d}$ is sufficiently smooth based on Assumption 3, (5) and (6) can be used to show that $\eta, \nu \in \mathcal{L}_{\infty}$. Property 2, Assumption 2 and (8) can be used to show that $\tau_{n} \in \mathcal{L}_{\infty}$. Let $\mathcal{S}_{\mathcal{D}} \subset \mathcal{D}$ denote the set defined as

$$
\mathcal{S}_{\mathcal{D}} \triangleq\left\{y \in \mathcal{D} \mid U_{2}(y)<\frac{1}{2} \min \{1, \underline{m}\} \rho^{-1}\left(2 \sqrt{\lambda_{1} k_{s}}\right)^{2}\right\} .
$$

The region of attraction in $\mathcal{S}_{\mathcal{D}}$ can be made arbitrarily large to include any initial conditions by increasing the control gain $k_{s}$. From (25), [45, Corollary 1] can be invoked to show that $c\|z(t)\|^{2} \rightarrow 0$ as $t \rightarrow \infty \forall y(0) \in \mathcal{S}_{\mathcal{D}}$. Based on the definition of $z$ in (15), $\left\|e_{1}(t)\right\| \rightarrow 0$ as $t \rightarrow \infty \forall y(0) \in \mathcal{S}_{\mathcal{D}}$.

\footnotetext{
${ }^{5}$ The $\operatorname{sgn}(\cdot)$ function can alternatively be defined as $\operatorname{sgn}(0)=0$; however, this restriction lacks robustness with respect to measurement noise. As described in results such as [41]-[43], Filippov's notion of a solution for discontinuous differential equations is appropriate to capture the possible closed-loop system behavior in the presence of arbitrarily small measurement noise. By utilizing the set valued map SGN $(\cdot)$ in the analysis, we account for the possibility that when the true state satisfies $x=0, \operatorname{sgn}(x)$ (of the measured state) falls within the set $[-1,1]$. Therefore, the presented analysis is more robust to measurement noise than an analysis that depends on sgn $(0)$ to be defined as a known singleton.

${ }^{6}$ The set of times $\Lambda \triangleq\left\{t \in[0, \infty): r(t)^{T} \beta K\left[\operatorname{sgn}\left(e_{2}(t)\right)\right]-r(t)^{T} \beta K\right.$ $\left.\left[\operatorname{sgn}\left(e_{2}(t)\right)\right] \neq 0\right\} \subset \mathbb{R}^{+}$is equivalent to the set of times $\left\{t: e_{2}(t)=\right.$ $0 \wedge r(t) \neq 0\}$. From (7), this set can also be represented by $\left\{t: e_{2}(t)=0 \wedge \dot{e}_{2}(t) \neq 0\right\}$. Provided $e_{2}(t)$ is continuously differentiable, it can be shown that the set of time instances $\left\{t: e_{2}(t)=0 \wedge \dot{e}_{2}(t) \neq 0\right\}$ is isolated, and thus, measure zero. This implies that the set $\Lambda$ is measure zero.
}

\section{REFERENCES}

[1] G. Griffiths, in Technology and Applications of Autonomous Underwater Vehicles, G. Griffiths, Ed. Boca Raton, FL, USA: CRC, 2003.

[2] M. Santhakumar and T. Asokan, "Coupled, non-linear control system design for autonomous underwater vehicle (AUV)," in Proc. Int. Conf. Control, Autom. Robot., Vis., 2008, pp. 2309-2313.

[3] S. Liu, D. Wang, and E. Poh, "Non-linear output feedback tracking control for AUVs in shallow wave disturbance condition," Int. J. Control, vol. 81, pp. 1806-1823, 2008.

[4] Z. Liu, "Reinforcement adaptive fuzzy control of wing rock phenomena," IET Control Theory Appl., vol. 152, pp. 615-620, 2005.

[5] M. Breivik and T. Fossen, "A unified control concept for autonomous underwater vehicles," presented at the Amer. Control Conf., Minneapolis, MN, USA, 2006.

[6] K. Do and J. Pan, "Robust and adaptive path following for underactuated autonomous underwater vehicles," in Proc. Amer. Control Conf., 2003, vol. 3, pp. 1994-1999.

[7] M. Jordan and J. Bustamante, "A speed-gradient adaptive control with state/disturbance observer for autonomous subaquatic vehicles," in Proc. IEEE Conf. Decision Control, 2006, pp. 2008-2013.

[8] R. P. Kumar, A. Dasgupta, and C. S. Kumar, "A new tracking controller design for underwater vehicles using quadratic stabilization," J. Dyn. Syst. Meas. Control, vol. 130, no. 2, pp. 024502-1-024502-6, 2008.

[9] L. Lapierre and B. Jouvencel, "Robust nonlinear path-following control of an AUV," IEEE J. Ocean. Eng., vol. 33, no. 2, pp. 89-102, Apr. 2008.

[10] K. Venugopal, R. Sudhakar, and A. Pandya, "On-line learning control of autonomous underwater vehicles using feedforward neural networks," IEEE J. Ocean. Eng., vol. 17, no. 4, pp. 308-319, Oct. 1992.

[11] M. Polycarpou, "Stable adaptive neural control scheme for nonlinear systems," IEEE Trans. Autom. Control, vol. 41, no. 3, pp. 447-451, Mar. 1996.

[12] J.-H. Li, P.-M. Lee, and Sang-Jeong Lee, "Neural net based nonlinear adaptive control for autonomous underwater vehicles," in Proc. IEEE Int. Conf. Robot. Autom., 2002, vol. 2, pp. 1075-1080.

[13] Y. Zhao, J. Farrell, and M. Polycarpou, "Localized adaptive bounds for online based control," in Proc. Amer. Control Conf., 2004, vol. 1, pp. 590 595.

[14] M. B. Cheng and C. C. Tsai, "Hybrid robust tracking control for a mobile manipulator via sliding-mode neural network," in Proc. IEEE Int. Conf. Mechatron., Taipei, Taiwan, 2005, pp. 537-542.

[15] E. Sebastian and M. A. Sotelo, "Adaptive fuzzy sliding mode controller for the kinematic variables of an underwater vehicle," J. Intell. Robot. Syst., vol. 49, no. 2, pp. 189-215, 2007.

[16] L.-J. Zhang, X. Qi, and Y.-J. Pang, "Adaptive output feedback control based on DRFNN for AUV,' IEEE J. Ocean. Eng., vol. 36, no. 9-10, pp. 716-722, Jul. 2009.

[17] A. Aguiar and J. Hespanha, "Trajectory-tracking and path-following of underactuated autonomous vehicles with parametric modeling uncertainty,' IEEE Trans. Autom. Control, vol. 52, no. 8, pp. 1362-1379, Aug. 2007.

[18] M. Corradini and G. Orlando, "A discrete adaptive variable-structure controller for MIMO systems, and its application to an underwater ROV,' IEEE Trans. Control Syst. Technol., vol. 5, no. 3, pp. 349-359, May 1997.

[19] D. Yoerger and J. Slotine, "Robust trajectory control of underwater vehicles," IEEE J. Ocean. Eng., vol. 10, no. 4, pp. 462-470, Oct. 1985.

[20] K. Goheen and E. Jefferys, "Multivariable self-tuning autopilots for autonomous and remotely operated underwater vehicles," IEEE J. Ocean. Eng., vol. 15, no. 3, pp. $144-151$, Jul. 1990.

[21] P.-M. Lee, S.-W. Hong, Y.-K. Lim, C.-M. Lee, B.-H. Jeon, and J.-W. Park, "Discrete-time quasi-sliding mode control of an autonomous underwater vehicle," IEEE J. Ocean. Eng., vol. 24, no. 3, pp. 388-395, Jul. 1999.

[22] G. Indiveri, M. Pino, M. Aicardi, and G. Casalino, "Nonlinear timeinvariant feedback control of an underactuated marine vehicle along a straight course," in Proc. IFAC Conf. Manoeuvr. Control Marine Craft, 2000, pp. 221-226.

[23] J. Kim, K. Kim, H. Choi, W. Seong, and K.-Y. Lee, "Estimation of hydrodynamic coefficients for an AUV using nonlinear observers," IEEE J. Ocean. Eng., vol. 27, no. 4, pp. 830-840, Oct. 2002.

[24] P. M. Patre, W. MacKunis, K. Kaiser, and W. E. Dixon, "Asymptotic tracking for uncertain dynamic systems via a multilayer neural network feedforward and RISE feedback control structure," IEEE Trans. Automat. Control, vol. 53, no. 9, pp. 2180-2185, Oct. 2008

[25] N. Fischer, S. Bhasin, and W. E. Dixon, "Nonlinear control of an autonomous underwater vehicle: A RISE-based approach," in Proc. Am. Control Conf., San Francisco, CA, USA, 2011, pp. 3972-3977. 
[26] T. I. Fossen, Handbook of Marine Craft Hydrodynamics and Motion Control. New York, NY, USA: Wiley, 2011.

[27] J. C. Kinsey, R. M. Eustice, and L. L. Whitcomb, "A survey of underwater vehicle navigation: Recent advances and new challenges," presented at the IFAC Conf. Manoeuvr. Control Marine Craft, Lisbon, Portugal, 2006.

[28] M.-C. Fang, P. Chang, and J.-H. Luo, "Wave effects on ascending and descending motions of the autonomous underater vehicle," Ocean Eng., vol. 33, pp. 1972-1999, 2006.

[29] L. Moreira and C. G. Soares, " $H_{2}$ and $H_{\infty}$ designs for diving and course control of an autonomous underwater vehicle in presence of waves," IEEE J. Ocean. Eng., vol. 33, no. 2, pp. 69-88, Apr. 2008.

[30] P. M. Patre, W. Mackunis, C. Makkar, and W. E. Dixon, "Asymptotic tracking for systems with structured and unstructured uncertainties," IEEE Trans. Control Syst. Technol., vol. 16, no. 2, pp. 373-379, Mar. 2008.

[31] B. Xian, D. M. Dawson, M. S. de Queiroz, and J. Chen, "A continuous asymptotic tracking control strategy for uncertain nonlinear systems," IEEE Trans. Autom. Control, vol. 49, no. 7, pp. 1206-1211, Jul. 2004.

[32] A. Filippov, "Differential equations with discontinuous right-hand side," Amer. Math. Soc. Transl., vol. 42, no. 2, pp. 199-231, 1964.

[33] A. F. Filippov, Differential Equations with Discontinuous Right-hand Sides. Norwell, MA, USA: Kluwer, 1988.

[34] G. V. Smirnov, Introduction to the Theory of Differential Inclusions. Providence, RI, USA: Amer. Math. Soc., 2002.

[35] J. P. Aubin and H. Frankowska, Set-Valued Analysis. Cambridge, MA, USA: Birkhäuser, 2008.

[36] D. Shevitz and B. Paden, "Lyapunov stability theory of nonsmooth systems," IEEE Trans. Autom. Control, vol. 39, no. 9, pp. 1910-1914, Sep. 1994.

[37] B. Paden and S. Sastry, "A calculus for computing Filippov's differential inclusion with application to the variable structure control of robot manipulators," IEEE Trans. Circuits Syst., vol. 34, no. 1, pp. 73-82, Jan. 1987.

[38] M. de Queiroz, J. Hu, D. Dawson, T. Burg, and S. Donepudi, "Adaptive position/force control of robot manipulators without velocity measurements: Theory and experimentation," IEEE Trans. Syst., Man, Cybern., vol. 27-B, no. 5, pp. 796-809, Sep. 1997.

[39] A. Hanai, H. T. Choi, S. K. Choi, and J. Yuh, "Experimental study on fine motion control of underwater robots," Adv. Robot., vol. 18, no. 10, pp. 963-978, 2004.

[40] F. H. Clarke, Optimization and Nonsmooth Analysis. Philadelphia, PA, USA: SIAM, 1990.

[41] H. Hermes, "Discontinuous vector fields and feedback control," in Differential Equations and Dynamical Systems. New York, NY, USA: Academic, 1967.

[42] J.-M. Coron and L. Rosier, "A relation between continuous time-varying and discontinuous feedback stabilization," J. Math. Syst. Estimation Contr ol, vol. 4, no. 1, pp. 67-84, 1994.

[43] R. Goebel, R. Sanfelice, and A. Teel, "Hybrid dynamical systems," IEEE Control Syst. Mag.,, vol. 29, no. 2, pp. 28-93, Apr. 2009.

[44] R. Leine and N. van de Wouw, "Non-smooth dynamical systems," in Stability and Convergence of Mechanical Systems with Unilateral Constraints (Lecture Notes in Applied and Computational Mechanics). Berlin, Germany: Springer-Verlag, 2008, vol. 36, pp. 59-77.

[45] N. Fischer, R. Kamalapurkar, and W. E. Dixon, "LaSalle-Yoshizawa corollaries for nonsmooth systems," IEEE Trans. Automat. Control, vol. 58, no. 9, pp. 2333-2338, Sep. 2013.

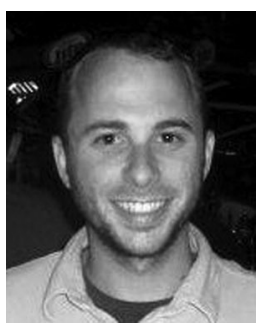

Nicholas Fischer received the Ph.D. degree in 2012 from the Department of Mechanical and Aerospace Engineering, University of Florida, Gainesville, FL, USA.

He is currently a Systems Engineer with Bluefin Robotics where his primary role includes simulation modeling, control architecture design, platform autonomy, and sensor integration of autonomous underwater vehicles. His research interests include the development of Lyapunov-based control methods for uncertain nonlinear systems and their application to

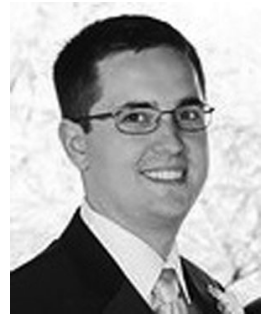

Devin Hughes received the M.S. degree in mechanical engineering in 2009 from the University of Florida, Gainesville, FL, USA, where he is currently working toward the Ph.D. degree.

His research interests include real-time path planning for underwater vehicles and control of automated machinery.

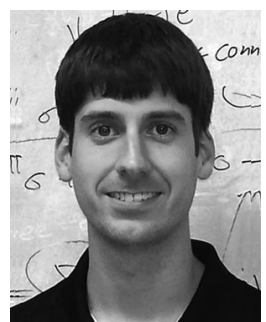

Patrick Walters received the M.S. degree in mechanical engineering from the University of Florida, Gainesville, FL, USA, in 2012, where he is currently working toward the Ph.D. degree with the Nonlinear Controls and Robotics Group.

His research interests include reinforcement learning-based feedback control, approximate dynamic programming, and robust control of uncertain nonlinear systems with a focus on the application of underwater vehicles. He also develops autonomous underwater vehicles with the Machine Intelligence Laboratory, University of Florida.

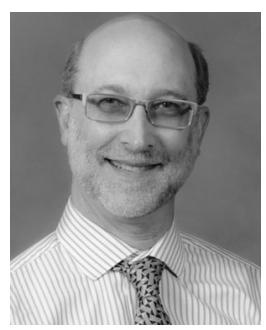

Eric M. Schwartz (SM'13) received the Ph.D. degree in 1995 from the Department of Electrical and Computer Engineering, University of Florida (UF), Gainesville, FL, USA.

$\mathrm{He}$ is a Master Lecturer with the Electrical and Computer Engineering Department, UF, and the Associate Director of the Machine Intelligence Laboratory (MIL). He is also the Director of several MIL robot teams including several competition teams. His robot submarines have competed for 16 years in the AUVSI Foundation and ONR's International RoboSub Competitions, with his SubjuGator teams winning three times and placing in the top 3 in 10 of the 16 years of this worldwide competition. His robot boat team, PropaGator, participated for the first time and won the 2013 AUVSI Foundation and ONR's International RoboBoat Competition. His MIL teams have also competed in several national lawnmower championships, winning in the static division once.

Dr. Schwartz has been the Advisor for the IEEE Student Branch and their robot team since 2001. He has also been the IEEE Gainesville Section Treasurer since 2001. He won the 2002-2003 UF Teacher of the Year award. In 20122013, he won the University of Florida IEEE and HKN 2012-2013 Teacher of the Year award.

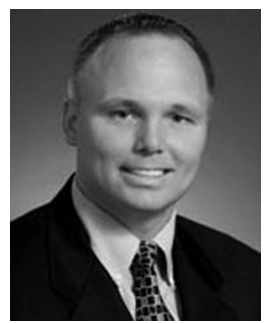

Warren E. Dixon (M'94-SM'05) received the Ph.D. degree in 2000 from the Department of Electrical and Computer Engineering, Clemson University, Clemson, SC, USA.

In 2004, he joined the Mechanical and Aerospace Engineering Department Faculty, University of Florida, Gainesville, FL, USA, where he is currently a Full Professor and a Charles Taylor Faculty Fellow (2012-2015) and where he holds a University of Florida Research Foundation Professorship (20122015). He has authored or coauthored three books, an edited collection, nine book chapters, and more than 250 refereed journal and conference papers. His main research interests include the development and application of Lyapunov-based control techniques for uncertain nonlinear systems.

Dr. Dixon served as an Appointed Member of the IEEE Control Systems Society Board of Governors (BoG) in 2008 and now serves as the Director of Operations for the Executive Committee of the BoG. He is currently or formerly an Associate Editor of the American Society of Mechanical Engineers (ASME) Automatica; the IEEE TRANSACTIONS ON SYSTEMS MAN AND CYBERNETICSPART B: CYBERNETICS; and the International Journal of Robust and Nonlinear Control. He received the 2011 ASME Dynamics Systems and Control Division Outstanding Young Investigator Award, the 2009 American Automatic Control Council O. Hugo Schuck Award, the 2006 IEEE Robotics and Automation Society Early Academic Career Award, the National Science Foundation CAREER Award (2006-2011), the 2004 U.S. Department of Energy Outstanding Mentor Award, and the 2001 Oak Ridge National Laboratory Early Career Award for Engineering Achievement. 